\title{
Síndrome de Sturge-Weber
}

\author{
Nelson Barbosa Franco Neto*, Fernanda Paula Franchini*, Vilson \\ Gentil Foletto Júnior**, Valdeci Juarez Pomblum***
}

RESUMO: A Síndrome de Sturge-Weber é uma doença rara, caracterizada por desordens cutâneas, neurológicas e oculares como conseqüência de malformações capilares faciais, angioma leptomeníngeo e anormalidades na vasculatura ocular. Dentre os achados clínicos mais comuns, evidencia-se proeminente mancha facial de cor semelhante ao vinho do Porto, convulsões e glaucoma. A Ressonância Nuclear Magnética é o padrão ouro na identificação de anormalidades estruturais encefálicas. $O$ tratamento foca as três principais manifestações da síndrome. $O$ laser corante pulsado permanece $o$ tratamento de escolha para a maioria das crianças com malformações capilares faciais. 0 tratamento padrão para o glaucoma tem sido a medicação continuada combinada com procedimentos cirúrgicos. $\mathrm{O}$ controle das convulsões é difícil e geralmente requer o uso de fármacos associados. Este trabalho objetiva expor aos clínicos as manifestações características e o tratamento desta Síndrome. Ainda são necessários esclarecimentos acerca da fisiopatologia da Síndrome, do estabelecimento de critérios diagnósticos e do tratamento.

Descritores: Síndrome de Sturge-Weber; Hemangioma; Glaucoma; Epilepsia.

\section{Sturge-Weber syndrome}

ABSTRACT: The Sturge-Weber syndrome is a rare disease, characterized by cutaneous, neurological and ocular disorders as a consequence of facial capillary malformations, leptomeningeal angiomatosis and ocular vasculature abnormalities. Amongst the most prevalent clinical findings are a prominent facial port-wine stain, glaucoma and seizures. The Magnetic Resonance Imaging has evolved as the gold standard for the identification of structural brain abnormalities. Treatment focuses on the three main manifestations of syndrome. Pulsed-dye laser remains the treatment of choice for most children with port-wine stains. The standard treatment for glaucoma has been the lifelong medical treatment in combination with surgical procedures. Seizure control is difficult and usually requires the administration of more than one drug. This work aims at to expose for clinicians the characteristic manifestations and treatment of this syndrome. Until the present, it is necessary lights concerning the physiopathology, the establishing diagnosis criteria and the treatment of the Syndrome.

Descriptors: Sturge-Weber Syndrome; Hemangioma; Glaucoma; Epilepsy.

\footnotetext{
*Acadêmicos do Curso de Graduação em Medicina da Universidade Federal de Santa Maria (UFSM), Santa Maria, RS, Brasil.

** Residente em Clínica Médica do Hospital Universitário de Santa Maria (HUSM), Santa Maria, RS, Brasil.

*** Professor Adjunto do Departamento de Clínica Médica da Universidade Federal de Santa Maria (UFSM), Santa Maria, Rio Grande do Sul, Brasil.
} 


\section{Introdução}

A Síndrome de Sturge-Weber (SSW) é uma síndrome congênita neurocutânea, esporádica, caracterizada por uma malformação capilar facial, um angioma leptomeníngeo ipsilateral e anormalidades na vasculatura ocular. Diferentemente de outras síndromes, a SSW não está associada a neoplasias intracranianas. Sua incidência é atualmente estimada em 1:50.000 nascidos vivos, apesar da identificação de formas intermediárias terem levado a um aumento dessa freqüência estimada ${ }^{1}$. Não há diferenças raciais ou de gênero. Casos familiares são raros - há apenas dois casos relatados na literatura ${ }^{2}$. Nessa síndrome, uma extensa malformação capilar-venosa afeta um ou, ocasionalmente, os dois hemisférios cerebrais; pode estar associada com epilepsia intratável, progressivo retardo mental e eventualmente requerer extensos procedimentos neurocirúrgicos como lobectomia ou hemisferectomia ${ }^{1}$.

\section{Metodologia}

Realizou-se um estudo do tipo exploratório descritivo com busca no banco de dados da Biblioteca Virtual de saúde do National Institute of Health - PUBMED, no mês de setembro de 2010. Os termos utilizados foram "Sturge-Weber" e "Sturge-Weber Syndrome". A pesquisa foi realizada pelo método integrado obtendo-se 950 publicações, sendo 129 artigos de revisão. A seleção das produções científicas desenvolveu-se a partir da leitura dos títulos e dos resumos. Utilizaram-se como critérios de seleção: a inclusão de artigos, textos completos, idiomas em português, espanhol e inglês Foram excluídos os trabalhos sem resumo, que não foram encontrados disponíveis na íntegra ou não se referiam especificamente ao tema.

\section{Manifestações clínicas}

As características primárias para o diagnóstico de SSW são uma malformação vascular cutânea facial e um angioma leptomeníngeo presentes desde o nascimento. Os componentes clínicos da Síndrome podem ser divididos em malformações cutâneas, neurológicas e oculares, sendo a mais proeminente uma mancha facial, usualmente unilateral, de cor semelhante ao vinho-do-porto (MVP), ipsilateral às malformações vasculares cerebrais. Todavia, nem todas as crianças com MVP possuem SSW. Esta malformação cutânea está presente em cerca de $0,3 \%$ de todos os nascidos vivos, sendo a malformação congênita vascular mais comum. Dentre as crianças com tal característica, aproximadamente $8 \%$ são portadoras da Síndrome ${ }^{3}$. Pode haver formas incompletas da SSW, de modo que algumas crianças a possuem sem, no entanto, apresentar MVP (5\% a 15\% dos casos), malformação ocular ou outros sintomas neurológicos ${ }^{1}$. Desta forma há três subdivisões clínicas da síndrome: a SSW tipo I, quando há malformações vasculares faciais e endocranianas com a possibilidade de glaucoma (forma clássica); a SSW tipo II, quando há angioma facial sem, no

Saúde (Santa Maria), Ahead of print, v.37, n.2, p. Sindromede entanto, evidência de envolvimento intracraniano; e a SSW tipo III, apenas com envolvimento leptomenínge ${ }^{4}$. 


\section{Manifestações cutâneas}

O portador da SSW, na grande maioria dos casos, apresenta um nevus angiomatoso congênito, geralmente observado na pálpebra superior e área supra-orbital, ipsilateral ao angioma leptomeníngeo. São manchas bem demarcadas e papulares que, microscopicamente, consistem em vasos sanguíneos hipertrofiados, sem evidência de hiperplasia, na derme e epiderme com progressivo espessamento e micro-nodulação múltipla, associados a cronicidade ${ }^{6}$. Poucas vezes, desenvolvem-se nódulos maiores que representam granulomas piogênicos ou tumores arteriovenosos acrais ${ }^{7}$. Geralmente as MVP não ultrapassam a linha média, entretanto, alguma extensão além deste limite pode ser observada ${ }^{3,5}$. As manchas sempre envolvem o território do primeiro ramo do nervo trigêmeo V1 - osso frontal, pálpebras ${ }^{3,5,8}$. Menos freqüentemente, também há envolvimento de V2 e V3, o que está associado a menor risco de malformações oculares e intracranianas 5 . Ainda, pode haver bilateralidade do nevus ( $10 \%$ a $30 \%$ dos casos $2,3,5)$. Acima de $20 \%$ dos pacientes apresentam envolvimento de V1 sozinho; a inclusão de V2 ou V2 e V3 está entre $2 \%$ a $23 \% 3,5,9$. O avanço da mancha para áreas maxilares e mandibulares está associado à hipertrofia de partes moles e crescimento excessivo das regiões ósseas subjacentes. Nesses casos é freqüente hiperplasia gengival e má erupção dos dentes ${ }^{10}$. A presença, ausência ou extensão da MVP não é proporcional à presença, grau e severidade dos sintomas neurológicos, nem é sua localização um indicador confiável da presença e topografia da lesão cerebral2,3,11.

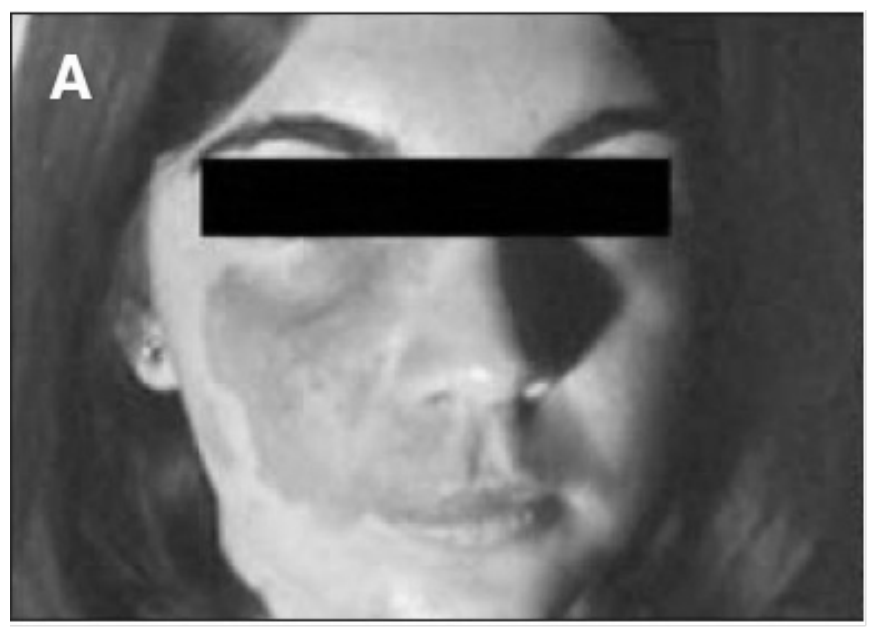

Figura 1 - Mancha de coloração vinhosa distribuída nos territórios V1 e V2. (extraído de Cronemberger et al33)

\section{Manifestações oculares}

Glaucoma é a manifestação oftalmológica mais comum na SSW, ocorrendo em 30\% a $70 \%$ dos pacientes ${ }^{9,12}$. É usualmente ipsilateral a MVP. Todavia, também pode ser bilateral a despeito de uma mancha unilateral ${ }^{13}$. O glaucoma pode ocorrer tanto na infância $(60 \%)$ quanto no adulto-jovem $(40 \%)^{12}$, estando presente, em média, por volta dos cinco anos ${ }^{9}, 0$ que implica necessidade de vigilância contínua. $O$ início precoce do glaucoma causa aumento dos diâmetros corneanos e miopia. 0 início tardio raramente conduzirá a tal desfecho ${ }^{12}$. A

fisiopatologia do glaucoma ainda não está totalmente elucidada, embora dois mecanismos Saúde (Santa Maria), Ahead of print, v.37, n.2, p. 09-17, 2011.

Franco Neto; Franchine; Foletto Júnior: tenham sido propostos. O glaucoma infantil estaria associado a anormalidades no seio 
camerular, o que aumentaria a resistência à saída do humor aquoso e, conseqüentemente, aumento da pressão intra-ocular. Em contraste, os pacientes, com início tardio do glaucoma, freqüentemente, possuem seio camerular normal ou com ligeiras anormalidades. Estes pacientes, entretanto, têm sinais clínicos de pressão venosa episcleral aumentada, causada por shunts arteriovenosos dentro do hemangioma episcleral levando a um possível aumento da pressão intra-ocular ${ }^{14}$. A segunda manifestação mais comum é 0 angioma coroidal, o qual pode contribuir para a causa do glaucoma. $O$ hemangioma é difuso, normalmente vermelho, plano a moderadamente elevado, produzindo uma aparência clássica de ketchup na fundoscopia. A exsudação contínua do tumor pode, cronicamente, levar a mudanças secundárias, tais como degeneração do epitélio pigmentário da retina, metaplasia fibrosa, degeneração cística e descolamento da retina, o que conduz a defeitos no campo e acuidade visual. 0 hemangioma coroidal sugere fortemente a presença de alterações na leptomeninge ${ }^{15}$. Outras manifestações oculares podem incluir dilatação e tortuosidade dos vasos conjuntivais e episclerais, heterocromia da íris, buftalmia, coloboma de disco óptico e catarata. Nevus de Ota pode coexistir ${ }^{16}$. Defeitos no campo visual podem ocorrer quando 0 córtex occipital está afetado.

\section{Manifestações neurológicas}

Uma das manifestações neurológicas mais comuns na SSW é a convulsão, ocorrendo em $23 \%$ a $83 \%$ dos pacientes ${ }^{2,9}$. A primeira manifestação convulsiva pode ocorrer entre 0 nascimento e 23 anos de idade, com uma média aos seis meses ${ }^{9}$. Os ataques tipicamente iniciam como episódios focais e, freqüentemente, são precipitados por febre e infecções ${ }^{17}$, podendo ser acompanhados ou seguidos pelo aparecimento de déficits focais motores tais como hemiparesia e hemiplegia ${ }^{2,17}$. Os sintomas podem ser focais, contralaterais a MVP, ou generalizados, com predomínio das formas focais. Convulsões generalizadas são, primeiramente, tônico-clônicas. Todavia, convulsões atônicas e crises de ausência também podem ocorrer ${ }^{2,11}$. Existe uma correlação direta entre a idade de início das convulsões, a extensão do envolvimento neurológico e 0 grau de deficiência intelectual ${ }^{17}$. Crianças sem epilepsia ou que iniciam os ataques depois dos quatro anos de idade têm inteligência e desenvolvimento normais ${ }^{9}$. 0 início precoce das convulsões pode estar relacionado a pior prognóstico e dificuldade no controle da epilepsia ${ }^{2}$. A ocorrência de convulsões prolongadas, durando mais de 60 minutos, correlaciona-se com epilepsia severa, déficits motores e deterioração mental ${ }^{11}$. Com o aumento da idade, as crises tendem a se tornar mais severas, freqüentes e complexas. Os mecanismos mais aceitos na patogênese das convulsões são a diminuição do fluxo sangüíneo cerebral nas áreas corticais envolvidas pela malformação vascular, bem como, a diminuição do retorno venoso, hipóxia focal e diminuição do metabolismo neuronal. Não obstante, registros eletrocorticográficos intra-operatórios têm demonstrado que a maior parte das áreas epileptogênicas ativas podem estar localizadas além das manchas angiomatosas ${ }^{18}$. A angiomatose leptomeníngea bilateral, presente em $7 \%$ a $26 \%$ dos pacientes, é fator de risco para convulsões mais severas, atraso no desenvolvimento e déficits focais. A malformação, usualmente, envolve os lobos occipital e

Saúde (Santa Maria), Ahead of print, v.37, n.2, p. 09-17, 2011. Sindrome de Sturge Weber parietal, mas pode afetar todo o hemisfério. Dilatação do plexo coróide é comum. Calcificações são observadas nas artérias meníngeas e veias corticais e subcorticais subjacentes ao angioma. Juntamente com a atrofia cortical, são consideradas uma 
consequêencia indireta de isquemia crônica, devido à estase vascular na área do angioma leptomenínge $0^{19}$. As anormalidades na vasculatura cerebral podem acarretar eventos hipóxicos, especialmente durante as crises convulsivas, quando a demanda de oxigênio é maior. Oclusão venosa e hipóxia podem contribuir para o declínio neurológico. Outras manifestações neurológicas incluem retardo mental, migrânea, déficits focais transitórios e déficit de atenção-hiperatividade. Cerca de $80 \%$ dos pacientes apresentam-se com problemas cognitivos $^{9}$. Clinicamente, a maioria das crianças atinge 0 marco de desenvolvimento apropriado para a idade nos primeiros meses de vida, mas aproximadamente metade dos pacientes com SSW não mantém esse padrão ${ }^{12}$. Dois terços exibem atraso psicomotor na infância ${ }^{2}$ e $50 \%$ tem significativo retardo mental tardio na idade escolar ${ }^{2,9}$. Enxaquecas iniciam em média aos oito anos de idade, sendo mais freqüentes em pessoas com déficits focais transitórios ${ }^{20}$. A enxaqueca secundária a SSW resulta, possivelmente, de um aumento no escoamento vasogênico de plasma e neuropeptídeos no espaço subaracnóide. Os neuropeptídeos podem ativar as fibras trigêmino-vasculares periféricas e produzir disfunção neuronal e vasodilatação no córtex correspondente ${ }^{21}$. Déficits focais transitórios caracterizamse por episódios de hemiparesia ou defeito de campo visual não diretamente associados à epilepsia. Os episódios podem durar de horas a vários dias ${ }^{22}$. No caso de hemiparesia, a extremidade envolvida pode não crescer com velocidade normal, resultando em hemiatrofia. A hipótese mais aceita para essa desordem é a ocorrência de trombose recorrente ${ }^{22}$. Outro modo comum de apresentação é o déficit de atenção-hiperatividade, que deve ser tratado clinicamente da mesma forma como se faz em pacientes com essa desordem, mas sem SSW, ou seja, monitoramento e administração de medicamentos que minimizem os sintomas ${ }^{17}$. Pode haver presença de hidrocefalia devido a um aumento da pressão venosa intracraniana causada por trombose de seios venosos profundos ou anastomoses arteriovenosas extensas ${ }^{23}$.

\section{Neuroimagem e eletroencefalografia}

A tomografia computadorizada (TC) e a ressonância nuclear magnética (RM) são os exames complementares mais úteis no diagnóstico da SSW, embora a TC por emissão de fóton único (SPECT), tomografia por emissão de pósitrons (PET) e espectroscopia por RM também tenham seu valor ${ }^{17}$. A TC é útil para demonstrar calcificações intracranianas, enquanto que a RM é válida para demonstrar o angioma leptomeníngeo ${ }^{24,25}$. $\mathrm{Na} \mathrm{TC}$, os achados incluem atenuação difusa da matéria branca superficial e profunda, presumivelmente devido a microcalcificações; realce giriforme após uso de contraste iodado sendo esta uma expressão da angiomatose pial; atrofia cerebral, como uma conseqüência de shunts vasculares nas estruturas corticais envolvidas pelo angioma; espessamento da caixa craniana, secundário à atrofia cerebra| ${ }^{26}$. A RM contrastada é o padrão-ouro para identificação de anormalidades na estrutura cerebral na SSW. A RM sem contraste pode mostrar atrofia cerebral, tanto na seqüência T1 quanto em T2, proeminência diplóica e aumento do plexo coróide. Calcificações podem ser observadas em T225. Após a injeção de gadolínio, há aumento pial difuso, visto nas imagens em T1. Esse é o critério isolado mais confiável para o diagnóstico de SSW. Aumento do plexo coróide do lado afetado e hemangioma coroidal são Saúde (Santa Maria), Ahead of print, v.37, n.2, p. 09-17, 2011.

Franco Neto; Franchine; Foletto Júnior; Pomblum. outros traços da SSW melhor observados na RM contrastada ${ }^{19}$. Apesar de não ser utilizada de 
forma usual, a angiografia por RM e a angiografia convencional mostram diminuição no calibre das artérias, dilatação das veias internas, aumento no tamanho do plexo coróide e ausência de veias hemisféricas superficiais do lado afetado ${ }^{24}$. A eletroencefalografia típica na SSW é assimétrica, com o hemisfério afetado mostrando uma redução na voltagem e lentificação do background. Essa assimetria pode ser detectada desde os primeiros meses de vida, todavia torna-se mais evidente quando a atrofia cerebral progride ${ }^{27}$.

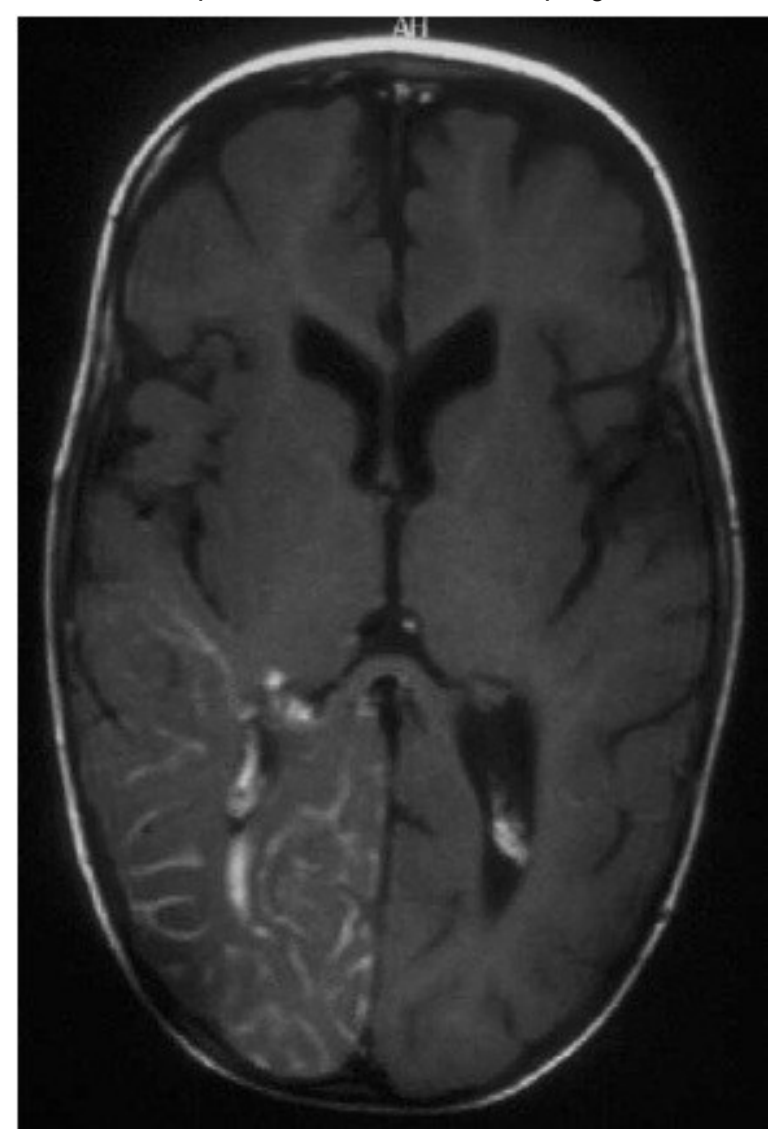

Figura 2 - Imagem por RM em T1 após injeção de gadolíneo mostrando realce do angioma leptomeníngeo no lobo occipital direito. (extraído de Baselga ${ }^{34}$ )

\section{Tratamento}

Freqüentemente, as modalidades de tratamento são focadas nas três principais manifestações da SSW: cutânea, ocular e neurológica. O laser corante pulsado permanece 0 tratamento de escolha para as MVP na maioria das crianças. A resposta no território de V1 nos pacientes com SSW é satisfatória, mas o resultado é menos exuberante quando existe concomitante envolvimento dos territórios de $\mathrm{V} 2$ e $\mathrm{V} 3^{28}$. 0 início precoce da terapia correlaciona-se com uma melhor resposta ${ }^{29}$. Fatores que influenciam na resposta ao tratamento são a profundidade e o diâmetro dos vasos sangüíneos constituintes da mancha ${ }^{30}$. Com o tempo, a lesão cresce e pode escurecer, desenvolvendo ectasias vasculares que promovem nodulações e vesículas superficiais se 0 tratamento com laser não for realizado ${ }^{31}$.

A terapia padrão para o glaucoma é o tratamento medicamentoso contínuo combinado com freqüentes cirurgias ${ }^{8}$, com o objetivo de controlar a pressão intra-ocular e prevenir danos ao nervo óptico. Apesar disso, o tratamento inicial é realizado apenas com as medicações como beta-bloqueadores e inibidores da anidrase carbônica ${ }^{32}$. Procedimentos cirúrgicos são 
realizados em pacientes que não respondem ao tratamento clínico, porém a anormalidade na vasculatura e a elevada pressão venosa episcleral reduzem as taxas de sucesso das cirurgias $^{8}$. Nos casos de grandes hemangiomas coroidais circunscritos, com fluido subretinal, o tratamento com placa de braquiterapia episcleral é freqüentemente considerado efetivo.

O tratamento da epilepsia é difícil e geralmente requer o uso de associação de fármaco. Carbamazepina ou oxcarbazepina são a primeira escolha, com fenobarbital e fenitoína como terapias de segunda linha. Se o controle não for alcançado, valproato de sódio ou topiramato são usados em associação com a carbamazepina. Reserva-se o uso de monoterapia com valproato ou topiramato como última tentativa ${ }^{17}$. Um controle ruim das crises convulsivas está relacionado à progressiva deterioração mental e déficit motor2. Uma variedade de procedimentos cirúrgicos tem sido proposta. Excisões corticais, lobectomia ou hemisferectomia são as mais freqüentes estratégias terapêuticas, devido ao caráter focal $\mathrm{e}$ unilateral na grande maioria dos casos. A literatura tem mostrado que quase todos os pacientes submetidos à lobectomia apresentaram diminuição da freqüência de convulsões, entretanto, poucos ficaram totalmente curados ${ }^{18}$. Em pacientes com convulsões severas, que não respondem à medicação, a hemisferectomia, pode ser uma opção, ainda que os riscos superem os benefícios ${ }^{8}$. Se a trombose for considerada uma causa da deterioração clínica, a terapia com ácido acetil-salicílico deve ser considerada. A aspirina pode ser usada também como prevenção de ataques isquêmicos transitórios, diminuindo, assim, sua freqüência ${ }^{22}$.

\section{Conclusão}

A despeito da quantidade de informação publicada sobre a SSW, muito ainda precisa ser esclarecido acerca da fisiopatologia - especialmente do glaucoma - sugerida nos modelos teóricos, os quais necessitam expansão. Ainda, o estabelecimento de critérios diagnósticos não está bem demonstrado na literatura. Novos ensaios clínicos são necessários para firmar melhor manejo e tratamento precoce nos casos de epilepsia com evolução para a intratabilidade e/ou desenvolvimento para déficit intelectual. As MVP requerem, da mesma forma, desenvolvimento de terapêutica mais efetiva.

\section{Referências}

1. Di Rocco C, Tamburrini G. Sturge-Weber Syndrome. Childs Nerv Syst, 2006;22:909-21.

2. Pascual-Castroviejo I, Diaz-Gonzalez C, Garcia-Melian RM, et al. Sturge-Weber syndrome. Study of 40 patients. Pediatr Neurol, 1983;9:283-8.

3. Tallman B, Tan OT, Marelli JG, et al. Location of port-wine stains and the likelihood of ophthalmic and/or central nervous system complications. Pediatrics, 1991;87:323-7.

4. Roach ES. Neurocutaneous syndromes. Pediatr Clin North Am, 1992; 39: 591-620.

5.Enjolras O, Riche MC, Merland JJ. Facial port-wine stains and Sturge-Weber syndrome. Pediatrics, 1985; $76: 48-51$.

Saúde (Santa Maria), Ahead of print, v.37, n.2, p.

6. Mulliken JB - Capillary (port-wine) and other telangiectatic stains, In: McAllister L ed. - Vascular Birthmarks. Pomblum Hemangiomas and Malformations. Philadelphia, Sauders, 1988;172-81. 
7. Carrasco L, Pastor A, Farina C, et al. Acral arteriovenous tumor developed within a nevus flammeus in a patient with Sturge-Weber syndrome. Am J Dermatol, 2003;25:341-5.

8. Bodensteiner JB, Roach ES. Sturge-Weber Syndrome. Mt. Freedom, NJ: The Sturge-Weber Foundation 1999.

9. Sujanski E, Conradi S. Outcome of Sturge-Weber syndrome in 52 adults. Am J Med Genet, 1995;57:35-45.

10. Ahluwalia TP, Lata J, Kanwa P. Sturge-Weber syndrome with intraoral manifestations. A case report. Indian J Dent Res, 1998;9:140-4.

11. Ville D, Enjolras O, Chiron C, et al. Prophilatic antiepileptic treatment in Sturge-Weber disease. Seizure, 2002;11:45-50.

12. Roach ES - Congenital cutaneovascular disorders, In: Bogouslavsky J, Caplan LR, eds - Stroke syndromes. London, Cambridge University Press, 1995;481-90.

13. Amirikia A, Scott IU, Murray TG. Bilateral diffuse choroidal hemangiomas with unilateral facial nevus flammeus in Sturge-Weber syndrome. Am J Ophthalmol, 2000;130:362-4.

14. Phelps CD. The pathogenesis of glaucoma in Sturge-Weber syndrome. Ophthalmology, 1978;85:276-86.

15. Madlom MM, Hoggard N, Griffths PD, et al. Facial naevus flammeus with choroidal haemangioma and without intracranial involvement. Dev Med Child Neurol, 2003;45:139.

16. Celebi S, Alagoz G, Aykan U. Ocular findings in Sturge-Weber syndrome. Eur J Ophthalmol, 2000;10:23943.

17. Thomas-Sohl KA, Vaslow DF, Maria BL. Sturge-Weber syndrome: a review. Pediatr Neurol, 2004;30(5):303-10.

18. Rasmussen TH, Mathieson G, Le Blanc F. Surgical therapy of typical and a forme fruste variety of SturgeWeber syndrome. Schweiz Arch Neurol Psychiatr, 1972;111:393-409.

19. Terdjman P, Aicardi J, Sainte-Rose C, et al. Neuroradiological findings in Sturge-Weber syndrome (SSW) and isolated pial angiomatosas. Neuropediatrics, 1991;22:115-20.

20. Kossoff EH, Hatfield LA, Ball KL, Comi AM. Comorbidity of epilepsy and headache in patients with SturgeWeber syndrome. J Child Neurol, 2005;20:678-82.

21. Lizuka T, Sakai F, Yamakawa K, et al. Vasogenic leakage and the mechanism of migraine with prolonged aura in Sturge-Weber syndrome. Cephalalgia, 2004;24:767-70.

22. Maria BL - Current management in child neurology. London, BC Decker, 2002;411-5.

23. Fishman MA, Baram TZ. Megalencephaly due to impaired cerebral venous return in Sturge-Weber variant syndrome. J Child Neurol, 1986; 1:115.

24. Vogl TJ, Stemmler J, Bergman C, et al. MR and MR angiography of Sturge-Weber syndrome. Am J Neuroradiol, 1993;14:417-25.

25. Vilela PF. Síndroma de Sturge Weber Revisitado: Avaliação das alterações morfológicas encefálicas por Tomografia Computadorizada e Ressonância magnética. Acta Med Port, 2003;16:141-8.

26. Griffiths PD. Sturge-Weber syndrome revisited: the role of neuroradiology. Neuropediatrics, 1996;27:28494.

27. Brenner RP, Sharbrough FW. Electencephalographic evaluation in Sturge-Weber syndrome. Neurology, 1976;26:629-32.

Saúde (Santa Maria), Ahead of print, v.37, n.2, p. 09-17, 2011.
28. Taïeb A, Touati L, Cony M. Treatment of port-wine stains with the 585-nm flashlamp-pulsed tunable dye laser: a study of 74 patients. Dermatology, 1994;188:276-81.

29. Batta K. Management of large birthmarks. Semin Neonatol, 2000;5:325-32. 
30. Lanigan SW. Port-wine stains unresponsive to pulsed dye laser: explanations and solutions. $\mathrm{Br} \mathrm{J}$ Dermatol, 1998;139:173-7.

31. Geronemus RG, Ashinoff R. The medical necessity of evaluation and treatment of port-wine stains. J Dermatol Surg Oncol, 1991;17:76-9.

32. Van Emelen C, Goethals M, Dralands L, et al. Treatment of glaucoma in children with Sturge-Weber syndrome. J Pediatr Ophthalmol Strabismus, 2000;37:29-34.

33. Cronemberger S, Calixto N, Assunção DAM, Milhomens EG. Síndrome de Sturge-Weber: estudo ultrabiomicroscópico. Arq Bras Oftalmol, 2004; 67: 211-7.

34. Baselga E. Sturge-Weber Syndrome. Semin Cut Med Surg, 2004; 23: 87-98.

\section{Nelson Barbosa Franco Neto}

Endereço para correspondência - Rua Erly de Almeida Lima 143, ap 301, CEP 97105-120, Santa Maria, RS, Brasil.

E-mail: nefneto@gmail.com

Currículo lattes: http://lattes.cnpq.br/7385270174358253

Recebido em 21 de março de 2011.

Aprovado em 26 de setembro de 2011. 
Saúde (Santa Maria), Ahead of print, v.37, n.2, p.

Sindrome de Sturge Weber

\begin{tabular}{l|l}
18 & ISSN 2236-5834
\end{tabular} 